
\title{
28 Research Square \\ Title MiR-188-5p Affects the Progression of Alzheimer's disease by Negatively Regulating Neuritin Expression
}

Jiawei Sun

Shihezi University School of Medicine

Dandan Song

Hangzhou Normal University

yin song

Jiaxing Center for Disease Control and Prevention

Guoxiang Li

No. 948th Army Hospital

Pingping Meng

Shihezi University School of Medicine

Jingling Zhu

Shihezi University School of Medicine

Jin Huang ( $\nabla$ huangjin623@163.com )

Shihezi University School of Medicine

\section{Research Article}

Keywords: Alzheimer's disease, Neuritin, microRNA-188-5p, bioinformatics, APP/PS1 mice

Posted Date: November 3rd, 2021

DOI: https://doi.org/10.21203/rs.3.rs-1025611/v1

License: (c) (1) This work is licensed under a Creative Commons Attribution 4.0 International License. Read Full License 


\section{Abstract}

\section{Background}

Alzheimer's disease (AD) is a progressive neurodegenerative disease characterized by complex pathobiological characteristics and still lacks accurate biomarkers in early diagnosis. Neuritin (NRN1) is a neurotrophic factor, which has active roles in neuronal plasticity and regeneration. Recent research suggests that Neuritin has been associated with loss of cognitive function, maybe a potential therapeutic target in $A D$.

\section{Methods}

We have clustered the upregulated miRNAs in $A D$ and predicted the target miRNAs of Neuritin by bioinformatics analysis, found miR-188-5p may involve in the development of AD.

\section{Results}

In the present study, we confirm the association between Neuritin and miR-188-5p expression in mice with AD. Besides, we provide evidence for the changes of cognition capacity, miR-188-5p and Neuritin levels in APP/PS1 mice.

Conclusion

Our results reveal a previously undefined mechanism that miR-188-5p plays a significant role in the development of $A D$ by inhibiting the expression of target protein Neuritin.

\section{Introduction}

Alzheimer's disease $(A D)$ is a progressive neurodegenerative disease characterized by the accumulation of amyloid $\beta$ plaques and by neurofibrillary tangles, with eventual progressive cognitive and memory impairment [1-3]. Due to its complex pathobiological characteristics, accurate biomarkers in early diagnostic and effective therapeutic options are still lacking in the detection or treatment of $A D[4,5]$.

Neuritin (NRN1) is a neurotrophic factor, which is also known as the candidate plasticity gene 15 (CPG15) [6]. Neuritin has multiple roles in neural development, including neuronal plasticity and regeneration $[7,8]$. Recent research suggests that Neuritin has been associated with loss of cognitive function, maybe a potential therapeutic target in AD [9]. However, the mechanisms of Neuritin in neurodegenerative diseases are not fully understood.

The further development of bioinformatics approaches has broadened the field of microRNA (miRNA) research, especially in target gene prediction and target site accessibility [10-12]. Studies have confirmed that miRNA dysregulation plays an important role in the development of a variety of diseases [13] and maybe involved as biomarkers in the progression of $A D$ [14-16]. 
Here, we clustered the up-regulated miRNAs in AD from the literature and datasets of AD and predicted the target miRNAs of Neuritin by bioinformatics analysis, found the association between miR-188-5p and Neuritin expression in mice model with AD (APP/PS1 mice). Also, we provided evidence for the associations between the cognition (working memory) capacity of APP/PS1 mice and altered miR-188-5p and Neuritin levels. Our results reveal a previously undefined mechanism that miR-188-5p plays a significant role in the development of $A D$ by inhibiting the expression of target protein Neuritin.

\section{Materials And Methods}

\section{Ethical statement}

All experiments using animals were performed in strict accordance with the Institutional Animal Care and Use Committee (IACUC) and approved by the Animal Ethical Committee of Hangzhou Normal University (Hangzhou, China).

\section{Animals}

APPswe/PS1 $\triangle E 9$ (APP/PS1) double-transgenic AD mice and age-matched B6C3 hybrid genetic wild-type (WT) mice were purchased from The Animal Model Center of Nanjing University (Nanjing, China).Mice were housed in same-sex groups and kept on a regular diet and light/dark cycle in a Specific Pathogen Free (SPF) laboratory animal environmental facilities, in which food and tap water were available ad libitum.Both APP/PS1 AD mice and WT control mice were divided into three age cohorts $₫ 1 \otimes 4$, and 7

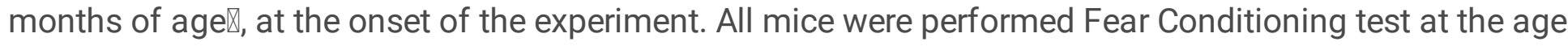
of 4 and 7 months and euthanized for hippocampus and cortex tissue extraction from the brain at the age of 1,4 , and 7 months.

RNA extraction and quantitative RT-PCR

The total RNA from the mice hippocampus and cortex was extracted by SanPerp Column microRNA Extraction Kit (Sangon, SK8191) and cDNAs were synthesized from RNA templates by RevertAid Premium Reverse Transcriptase (Thermo ScientificTM, EP0733). The reverse transcription products were amplified by SG Fast qPCR Master Mix (2X, Roche, B639271). The neuritin expression from the mice hippocampus and cortex was evaluated by qPCR using FastKing gDNA Dispelling RT SuperMix Kit (TIANGEN, KR170801). The extraction of total RNA and synthesis of cDNAs was performed with TRIzolTM reagent (Invitrogen, 15596026) according to the manufacturer's protocol. The A260/A280 ratio was recorded and the concentration and the purity of RNA were assessed by Nanodrop 2000. The expression levels of miRNA and genes were normalized to U6 snRNA levels and GAPDH, respectively.

The primers were designed by the Primer Premier 5.0 Software and as follows:

miR-188-5p-F: 5'-ACACTCCAGCTGGGCATCCCTTGCATGG-3'

miR-188-5p-R: 5'-CTCAACTGGTGTCGTGGAGTCGGCAATTCAGTTGAGCCCTCCA-3' 
U6-F: 5'-CTCGCTTCGGCAGCACA-3'

U6-R: 5'-AACGCTTCACGAATTTGCGT-3'

neuritin-F: 5'-ATTTCACTGATCCTCGCGGT-3'

neuritin-R: 5'-CCAGTATGTGCACACGGTCT-3'

GAPDH-F: 5'-CAGGAGAGTGTTTCCTCGTCC-3'

GAPDH-R: 5'-TTCCCATTCTCGGCCTTGAC-3'

Western Blot assay

The Western Blot assay was used to determine the expression of Neuritin protein in hippocampal tissues, cortical tissues, and 293T cells. The samples were collected on ice and lysed in RIPA lysis buffer (with 1\% PMSF) for 45 minutes at $4^{\circ} \mathrm{C}$, centrifuged at $12,000 \mathrm{r} / \mathrm{min}$ for $10 \mathrm{~min}$ at $4^{\circ} \mathrm{C}$, and the supernatant was collected. The concentration of protein was measured by BCA Protein Assay Kit (TIANGEN, PA115-01). Equal amounts of protein $(30 \mu \mathrm{g})$ were separated by $12.5 \%$ SDS-PAGE and transferred onto a PVDF membrane by semi-dry electrophoretic transfer. After 2 hours of blocking, the PVDF membrane was probed with the rabbit anti-Neuritin (Abcam, ab64186, 1:500 dilution) and the mouse anti- $\beta$-actin (ZSGBBIO, TA-09, 1:1000 dilution) for more than 12 hours at $4^{\circ} \mathrm{C}$. Then, incubated the washed PVDF membrane with the matched secondary antibody with HRP for 2 hours at room temperature. The bands were visualized with an enhanced chemiluminescence system and protein levels of Neuritin were normalized to the bands of $\beta$-actin.

Bioinformatics analysis of candidate miRNAs

To obtain miRNA datasets, by searching for miRNA expression profiling data in AD from the Gene Expression Omnibus database (http://www.ncbi.nlm.nih.gov/geo/), using R language to select criteria samples from $A D$ and NC groups, excluding epigenetic data. Candidate miRNAs were screened from 3 datasets of AD (GSE46579, GSE104514, and GSE16759), by a matrix of miRNA expression levels in the Bioconductor LIMMA R package. The screening threshold of candidate miRNAs was $P<0.05$, Ifold change $\mid>1$.

To predict the miRNAs that target Neuritin, we used 4 bioinformatics algorithms including TargetScan (http://www.targetscan.org/), miRBD (http://mirdb.org/), DIANA-microT (http://diana.imis.athenainnovation.gr/) and miRWalk (http://mirwalk.umm.uni-heidelberg.de/). named VENNY (https://bioinfogp.cnb.csic.es/tools/venny/index.html), an online Venn diagram software, was used to screen miRNAs that were both differentially expressed in $A D$ and that target Neuritin.

All homo sapiens and mice sequences related to candidate RNAs were retrieved from miRBase (http://www.mirbase.org/), multiple sequence alignment analysis, and phylogenetic tree construction were performed by EMBL-Cluster Omega (http://www.ebi.ac.uk/Tools/msa) and Jalveiw software. 
Fear Conditioning (FC) test was taken place in 2 chambers (Panlab, LE116, Barcelona, Spain) as described [17-20]. There was a speaker attached to one of the stainless-steel walls in the chambers, which provided continuous background noise $(30 \mathrm{~dB})$ during the test. The stainless-steel grid floor of the chamber could deliver electric foot shocks, from which the mice could not escape. The 4- and 7- monthsold APP/PS1 AD mice ( $N=5$ per age cohort) and age-matched WT mice ( $N=5$ per age cohort) were tested in the whole FC test.

The FC test consisted of 2 phases, the training and the test phase, which took place in 48 consecutive hours. During the training phase, all groups of mice were placed in a standard fear conditioning chamber A $(25 \times 25 \times 25 \mathrm{~cm})$ and allowed to acclimate for 120 seconds without stimulation. After acclimation, each mouse underwent 7 cycles of tones ( 30 seconds per cycle, $80 \mathrm{~dB}$ ) and electric foot shocks ( 2 seconds duration per cycle, $0.35 \mathrm{~mA}$ ), which stopped with the tones. Each foot shock was separated by a 30-60 seconds time period. The training phase ended with a 30 seconds post-training period, then mice were removed from the chamber and returned to the home cages. During the test phase, the mice underwent fear conditioning testing for both the contextual and cued memory tests, at 3, 24, and 48 hours after the training phase. For the contextual recall test, the mice were placed back in chamber $A$ without any tones and foot shocks for 6 minutes. For the cued recall test, the mice were placed in chamber $B(29 \times 25 \times 22 \mathrm{~cm}$, with different walls, floor, smell, and light, compared with chamber A), treated with the same acclimation, tones, and post-training period as the training phase, without foot shocks. For each phase and test, the freezing behavior, which is defined as the cessation of all movements despite respiratory behavior [21], was recorded. The percentage of the time that the mice displayed freezing behavior was calculated and the differences between groups in time spent freezing were analyzed.

\section{Cell culture and transfection}

The 293T cells were cultured, as described previously [22], in Dulbecco's Modified Eagle's Medium (DMEM) (Sigma, D0819, UK) containing 10\% heat-inactivated Fetal Bovine Serum (FBS) (Biological Industries, $04-001-1 \mathrm{ACS}$, Israel), in $37^{\circ} \mathrm{C}$ in a humidified atmosphere of $5 \% \mathrm{CO}_{2}$.

The 293T cells were transfected using miR-188-5p mimics or normal control (NC) miRNA and treated for 24 hours. Then, the cells were collected for later experiments.

\section{Luciferase assay}

A luciferase activity assay was performed using a Dual-Luciferase Reporter Assay Kit (Promega, USA), according to the manufacturer's instructions.

The 293T cells were seeded in 24-well plates for 12 hours and divided into 3 groups, which were cotransfected with miR-188-5p mimics and promoter-luciferase constructs normal control (pLUC-NC), miR188-5p mimics and pLUC-neuritin mutant (pLUC-MUT-neuritin), or miR-188-5p mimics and pLUC-neuritin 
by using Lipofectamine 3000 reagent (Invitrogen, Grand Island) according to the manufacturer's instructions. Luciferase activity was analyzed 12 hours after transfection by using the Dual-Luciferase Reporter Assay System.

The primers were designed and applied as follows:

pLUC-neuritin-F: 5'-GTCATAAGAGATGCTTCTAGAGTCGACCTGCAG -3'

pLUC-neuritin-R: 5'-AGCATCTCTTATGACTTTAAACAACTAGAATTA-3'

pLUC-MUT-neuritin-F: 5'-GTCCTAUGAAUAGCTTCTAGAGTCGACCTGCAG-3'

pLUC-MUT-neuritin-R: 5'-AGCTATTCATAGGACTTTAAACAACTAGAATTA-3'

Statistical analysis

Bioinformatics analysis was performed using R software. Statistical analysis was performed using GraphPad 6.0. Differences between two groups were determined using one-way analysis of variance (ANOVA), and multiple comparisons were performed with a t-test posthoc test. All values were presented as the mean \pm standard deviation (SD). $P<0.05$ was considered statistically significant.

\section{Results}

\section{Decrease of Neuritin expression in the hippocampus of APP/PS1 mice.}

In the hippocampus and cortex of APP/PS1 mice at 1, 4, and 7 months, the expression of Neuritin mRNA and protein level was evaluated by qPCR analysis and Western blot (Fig. 1). In accordance with the result of qPCR, the expression of Neuritin mRNA was notably lower in the hippocampus of the APP/PS1 mice, compared with the WT mice. And, the Neuritin mRNA expression in the hippocampus of APP/PS1 mice at the lowest level in 4-months old $(P<0.01$, Fig. 1 A). Similarly, the Western blot analysis showed significant reductions in the expression level of the Neuritin protein in the hippocampus at 4- and 7-months old APP/PS1 mice, compared with the age-matched WT mice ( $P<0.05$, Fig. $1 \mathrm{C}, \mathrm{D})$. However, no group differences were found in the expression level of Neuritin mRNA in cortexes at each age group of mice (Fig.1 B), nor were differences observed in the expression level of Neuritin protein in the hippocampus of 1-month-old APP/PS1 mice, compared with the WT mice (Fig.3 A, C, D). Revealing that Neuritin level was downregulated in the hippocampus of APP/PS1 mice from the age of 4 months.

\section{The selection of candidate miRNAs that may regulate Neuritin in AD by Bioinformatics.}

We collected 3 miRNA expression datasets (GSE46579, GSE104514, and GSE16759) from AD patients and $A D$ model mice as well as literature data. $R$ language conductor package was used to normalize the measurement data, standardize the quality and cluster analysis of the 3 datasets. MiRNAs were screened using the cut-off criteria of $P$-value $<0.05$ and $|\log 2 \mathrm{FC}| \geq 1$ by the LIMMA R package. According to the volcano plots, 96 and 51 miRNAs were up-regulated in GSE46579 (Fig. 2A) and GSE104514 (Fig. 2B), 
respectively. Candidate miRNAs (miR-188 and miR-184) were obtained by overlapping up-regulated miRNAs (Fig. 2C).

We further searched for the candidate miRNAs that may target the 3'-UTR of Neuritin by 4 bioinformatics algorithms. 481 miRNAs from TargetScan, 92 miRNAs from miRBD, 253 miRNAs from DIANA-microT, and 58 miRNAs from miRWalk were overlapped by VENNY (Fig. 2D). We selected 7 miRNAs target the Neuritin for the candidate miRNAs.

Then, we performed multiple sequence alignment and homology analysis of candidate miRNAs from different species with bioinformatics websites and software (Fig. 2E). The phylogenetic tree showed the homology of miR-188-5p and miR-188-3p in Homo sapiens and mice (Fig. 2F, Green texts), and the global alignment of mmu-miR-188-5p and has-miR-188-5p sequences showed the identity (Fig. 2G).

\section{The variations of the candidate miRNAs (miR-188-5p) and Neuritin in the hippocampus of APP/PS1 mice.}

We investigated the expression of candidate miRNAs (miR-188-5p) in the hippocampus and cerebral cortexes of the APP/PS1 mice at the ages of 1,4 , and 7 months. MiR-188-5p was highly expressed in the hippocampus in 4 months of APP/PS1 mice $(P<0.05$, Fig. 3A). In addition, the assays showed that the expression of miR-188-5p was higher in 4 months than 1 and 7 months in the hippocampus of APP/PS1 mice, compared with the WT mice (Fig. 3C). However, the expression level of miR-188-5p in the cerebral cortex was not statistically significant, either in the APP/PS1 mice or compared with the WT mice (Fig. 3B). Furthermore, we detected the expression of Neuritin protein from the hippocampus of these mice, Western bolting assay showed the protein level of Neuritin decreased from 4 months in the APP/PS1 mice, compared with WT mice $(P<0.05$, Fig. 3D, E).

\section{Evaluation of cognition (working memory) capacity in APP/PS1 mice by Fear Conditioning test.}

Fear Conditioning test was performed at the age of 4 and 7 months for APP/PS1 and WT mice (Fig. 4A). In the training phase, in which we characterized the exploration of the training chamber ( 120 seconds) and the presentation of a tone (30 seconds, 7 times), no statistically differences were observed in the freezing times during the response to the administration of foot shock ( 2 seconds, $0.35 \mathrm{~mA}$ ) between the genotypes and age groups (Fig. 4B), suggesting that APP/PS1 and WT mice showed comparable initial reactivity to experimental stimuli.

In the test phase, mice underwent fear conditioning testing for both the contextual and cued recall. The analysis of the freezing times indicated that APP/PS1 mice at the age of 4 and 7 months spent less time freezing during the contextual fear memory conditioning, showed statistical differences at 3 and 48 hours after the training phase, compared with age-matched WT mice (Fig.4 C, E). On this basis, during the cued fear memory conditioning, a significant reduction of freezing times was found in response in APP/PS1 mice of different ages, compared with WT mice (Fig.4 D, F). The overall performance of the mice in the 
fear conditioning test revealed a significant decline in cognition capacity (working memory) of the APP/PS1 mice from the age of 4 months.

\section{MiR-188-5p down-regulated the expression of Neuritin in 293T cells.}

To validate the interaction of miR-188-5p with neuritin, we used the luciferase reporter constructs inserted with the 3'-UTR of neuritin and MUT-neuritin in 293T cells (Fig.5 A, B). As shown in Fig.5 C, the luciferase activity of neuritin 3'-UTR was higher than MUT-neuritin 3'-UTR, compared with the NC control and NC mimics $(P<0.001)$.

To further identify the regulatory role of miR-188-5p in Neuritin expression, 293T cells were transfected with miR-188-5p mimics. Results from Western Blot analysis showed that, in protein level (Fig. 5 D, E), the expression of Neuritin was significantly lower than NC mimics $(P<0.05)$. Therefore, miR-188-5p could down-regulate the expression of Neuritin by binding to the neuritin 3'-UTR in 293T cells.

\section{Discussion}

$A D$ is a highly heterogeneous condition, the neuropathologic criteria are the presence of amyloid- $\beta$ deposition and accumulation of hyperphosphorylated Tau, individuals with these pathological biomarkers may present with various degrees of cognitive impairment [23-25]. Neuritin is a neurotrophic factor activated by neurotrophins [26, 27], including synaptic plasticity [28, 29] and neurite outgrowth [3032]. Previous work has suggested that Neuritin against AD-related neural and cognitive defects $[9,33]$.

To evaluate the role of Neuritin in AD, we compared the expression level of Neuritin in the hippocampus of APP/PS1 mice at 1, 4, and 7 months with month-matched WT mice (Fig. 1). The assay at the mRNA level showed that, in all three month-groups, Neuritin was significantly reduced compared with WT mice in the hippocampus (Fig. 1 A), especially at 4 months which was regarded as a sub-clinical or early-clinical stage of $A D[34,35]$. Similarly, the Western blot analysis showed that the expression level of the Neuritin protein was consistent with the observation at the Neuritin mRNA level (Fig. 1C, D).

MiRNAs are widely evidenced as key regulators of functions such as neurite outgrowth [36], dendritic spine morphology [37], and synaptic plasticity [38]. At present, functions and mechanisms of miRNAs in $A D$ are increasingly recognized, which finding evidence about candidate miRNAs as biomarkers [39-42]. In order to explore the role of miRNAs and Neuritin in the pathogenesis of AD. We first performed bioinformatics analysis in published datasets to cluster 2 candidate miRNAs that up-regulated in AD (Fig. 2C). Secondly, 4 miRNA target gene prediction websites with different algorithms were used to overlap 7 candidate miRNAs that may target the 3'-UTR of neuritin (Fig. 2D). Thirdly, multiple sequence homology analysis (Fig. 2E) and phylogenetic tree (Fig. 2F) of candidate miRNAs showed the identity of mmu-miR188-5p and has-miR-188-5p, which corresponded to our previous studies [43], indicating a potential role of miR-188-5p in the pathogenesis of AD. 
To further confirm the bioinformatics results, we detected the expression levels of miR-188-5p in the hippocampus and cerebral cortexes of the APP/PS1 mice. Consistent with the bioinformatics analysis, we found miR-188-5p is significantly high-expressed in the hippocampus, which plays one of the most essential roles in learning and memory [44], in 4 months APP/PS1 mice, compared with WT mice (Fig. $3 A)$. Meanwhile, we detected the expression of Neuritin protein from the hippocampus of the same batch of mice (Fig. 3D). Interestingly, the expression level of miR-188-5p increased along with the decrease of the expression of Neuritin in the hippocampus at 4 months APP/PS1 mice (Fig. 3C, E). Revealing that change of Neuritin and miR-188-5p may be related to the genesis and development of AD.

Fear conditioning test is commonly used as a model for studying emotional learning and working memory in mice [45]. With the different brain circuitries of cognitive paradigms [46], we chose contextual and cued memory tests to evaluate the hippocampus-dependent [47] and amygdala dependent [48] memory in APP/PS1 mice (Fig. 4). The analysis of the freezing times from contextual and cued fear memory conditioning indicated that significant cognitive impairment occurred at the of 4 months and lasted until 7 months in APP/PS1 mice (Fig. 4C-F), notably in Short-term memory (STM) (Fig. 4B, $P<0.001)$. It is well established that the earliest detectable deficits in cognition and neuropathological changes of $A D$ are seen in the hippocampus, consistent with the progression of pathology $[49,50]$. Therefore, the results indicated that, with the appearance of cognitive impairment, the expression of Neuritin decreased, on the contrary, the expression of miR-188-5p increased, suggesting that miR-188-5p may be regulated the expression of Neuritin in the hippocampus of APP/PS1 mice.

Further, with the foundation data of the Fear conditioning test, we sought to determine the underlying mechanism of miR-188-5p, investigating whether miR-188-5p regulates the expression of Neuritin (Fig. 5). The luciferase reporter for Neuritin 3'-UTR was significantly inhibited by miR-188-5p (Fig. 5C), we got the further verification from the western blot of miR-188-5p mimics significantly inhibited the expression of Neuritin in 293T cells (Fig. 5D, E), further suggesting miR-188-5p may promote cognitive impairment through down-regulating the expression of Neuritin in the development of AD.

\section{Conclusion}

In summary, our study revealed a previously undefined mechanism that miR-188-5p plays a significant role in the development of $A D$ by inhibiting the expression of target protein Neuritin. These early alterations in miRNA and target protein in the hippocampus could be regarded as potential candidate biomarkers of AD. The effect of miR-188-5p and Neuritin on synaptic plasticity and pathology changes in $A D$ needs to be explored in our future studies.

\section{Abbreviations}




\begin{tabular}{|ll|}
\hline abbreviation & Full name \\
\hline AD & Alzheimer's disease \\
\hline NRN1 & Neuritin \\
\hline CPG15 & candidate plasticity gene 15 \\
\hline IACUC & Institutional Animal Care and Use Committee \\
\hline WT & wild-type \\
\hline SPF & Specific Pathogen Free \\
\hline FC & Fear Conditioning \\
\hline DMEM & Dulbecco's Modified Eagle's Medium \\
\hline FBS & Fetal Bovine Serum \\
\hline NC & normal control \\
\hline
\end{tabular}

\section{Declarations}

Funding: The present study was supported by the Natural Science Foundation of China (grant no. 81771173 to $\mathrm{JH}$ ) and the Zhejiang Provincial Natural Science Foundation of China (grant no. LY18H260002 to YH).

Author contributions: JH, JZ and DS contributed to the conception or design of the study. YS and JS contributed to the acquisition, analysis, or interpretation of all cell-level data. GL and JS contributed to the acquisition, analysis, or interpretation of the AD mouse data. YS contributed to the acquisition, analysis and interpretation of the bioinformatics data. PM contributed to the construction of the vectors. JS and $\mathrm{JH}$ contributed to the drafting of the the article and revising it critically for all content. All authors contributed to revising the work critically for important intellectual content.

Competing interests: The authors declare that they have no competing interests.

Availability of data and materials: The data used and/or analyzed during the present study are available from the corresponding author on reasonable request.

Conflicts of interest: All experimental procedures were approved by the Animal Ethics Committee of Hangzhou Normal University.

Consent for publication: All participants provided written informed consent at enrolment including consent to publication.

Statement: Our research accord to the ARRIVE guidelines (PLoS Bio 8(6), e1000412,2010). 
Acknowledgements The authors would like to thank the Animal Center of Hangzhou Normal University for breeding the mice. Dr Lei Yang (Department of Prevention Medicine, School of Medicine, Hangzhou Normal University, Hangzhou, Zhejiang 310036, P.R. China) kindly provided the experimental equipment.

\section{References}

[1] Masters, C.L., et al., Alzheimer's disease. Nature reviews. Disease primers, 2015. 1: p. 15056.

[2] Meldolesi, J., Alzheimer's disease: Key developments support promising perspectives for therapy. Pharmacological research, 2019. 146: p. 104316.

[3] Scheltens, P., et al., Alzheimer's disease. Lancet (London, England), 2016. 388(10043): p. 505-517. [4] Long, J.M. and D.M. Holtzman, Alzheimer Disease: An Update on Pathobiology and Treatment Strategies. Cell, 2019. 179(2): p. 312-339.

[5] van der Kant, R., L.S.B. Goldstein, and R. Ossenkoppele, Amyloid- $\beta$-independent regulators of tau pathology in Alzheimer disease. Nature reviews. Neuroscience, 2020. 21(1): p. 21-35.

[6] Yao, J.-J., et al., Functions and the related signaling pathways of the neurotrophic factor neuritin. Acta pharmacologica Sinica, 2018. 39(9): p. 1414-1420.

[7] Lu, J.-M., et al., Neuritin Enhances Synaptic Transmission in Medial Prefrontal Cortex in Mice by Increasing CaV3.3 Surface Expression. Cerebral cortex (New York, N.Y. : 1991), 2017. 27(7): p. 3842-3855.

[8] Zhang, P., et al., Neuritin Inhibits Notch Signaling through Interacted with Neuralized to Promote the Neurite Growth. Frontiers in molecular neuroscience, 2017. 10: p. 179.

[9] An, K., et al., Neuritin can normalize neural deficits of Alzheimer's disease. Cell death \& disease, 2014. 5: p. e1523.

[10] Chen, L., et al., Trends in the development of miRNA bioinformatics tools. Brief Bioinform, 2019. 20(5): p. 1836-1852.

[11] Hammond, S.M., An overview of microRNAs. Adv Drug Deliv Rev, 2015. 87: p. 3-14.

[12] Riffo-Campos, A.L., I. Riquelme, and P. Brebi-Mieville, Tools for Sequence-Based miRNA Target Prediction: What to Choose? Int J Mol Sci, 2016. 17(12).

[13] Rupaimoole, R. and F.J. Slack, MicroRNA therapeutics: towards a new era for the management of cancer and other diseases. Nat Rev Drug Discov, 2017. 16(3): p. 203-222.

[14] Cheng, L., et al., Prognostic serum miRNA biomarkers associated with Alzheimer's disease shows concordance with neuropsychological and neuroimaging assessment. Mol Psychiatry, 2015. 20(10): p. 1188-96. 
[15] Gupta, P., et al., miRNAs in Alzheimer Disease - A Therapeutic Perspective. Curr Alzheimer Res, 2017. 14(11): p. 1198-1206.

[16] Swarbrick, S., et al., Systematic Review of miRNA as Biomarkers in Alzheimer's Disease. Mol Neurobiol, 2019. 56(9): p. 6156-6167.

[17] Huff, N.C., Amygdala Regulation of Immediate-Early Gene Expression in the Hippocampus Induced by Contextual Fear Conditioning. Journal of Neuroscience, 2006. 26(5): p. 1616-1623.

[18] Izquierdo, I., C.R. Furini, and J.C. Myskiw, Fear Memory. Physiol Rev, 2016. 96(2): p. 695-750.

[19] Janus, C., et al., Behavioral abnormalities in APPSwe/PS1dE9 mouse model of AD-like pathology: comparative analysis across multiple behavioral domains. Neurobiol Aging, 2015. 36(9): p. 2519-32.

[20] Li, D.W., et al., Fear conditioning downregulates miR-138 expression in the hippocampus to facilitate the formation of fear memory. Neuroreport, 2018. 29(16): p. 1418-1424.

[21] Sacchetti, B., et al., Auditory thalamus, dorsal hippocampus, basolateral amygdala, and perirhinal cortex role in the consolidation of conditioned freezing to context and to acoustic conditioned stimulus in the rat. The Journal of neuroscience : the official journal of the Society for Neuroscience, 1999. 19(21): $p$. 9570-9578.

[22] Zhang, P., et al., Neuritin Inhibits Notch Signaling through Interacted with Neuralized to Promote the Neurite Growth. Front Mol Neurosci, 2017. 10: p. 179.

[23] Duyckaerts, C., B. Delatour, and M.C. Potier, Classification and basic pathology of Alzheimer disease. Acta Neuropathologica, 2009. 118(1): p. 5-36.

[24] Gross, A.L., et al., Alzheimer's disease severity, objectively determined and measured. Alzheimer's \& dementia (Amsterdam, Netherlands), 2016. 4: p. 159-168.

[25] ladanza, M.G., et al., A new era for understanding amyloid structures and disease. Nature reviews. Molecular cell biology, 2018. 19(12): p. 755-773.

[26] Naeve, G.S., et al., Neuritin: a gene induced by neural activity and neurotrophins that promotes neuritogenesis. Proceedings of the National Academy of Sciences of the United States of America, 1997. 94(6): p. 2648-2653.

27. Nedivi, E., et al., Numerous candidate plasticity-related genes revealed by differential cDNA cloning. Nature, 1993. 363(6431): p. 718-722.

28. Cantallops, I. and H.T. Cline, Rapid activity-dependent delivery of the neurotrophic protein CPG15 to the axon surface of neurons in intact Xenopus tadpoles. Developmental neurobiology, 2008. 68(6): p. 744-759. 
29. Subramanian, J., et al., CPG15/Neuritin Mimics Experience in Selecting Excitatory Synapses for Stabilization by Facilitating PSD95 Recruitment. Cell reports, 2019. 28(6).

30. Gao, R., et al., Exogenous Neuritin Promotes Nerve Regeneration After Acute Spinal Cord Injury in Rats. Human gene therapy, 2016. 27(7): p. 544-554.

31. Wang, H., et al., Recombinant hNeuritin Promotes Structural and Functional Recovery of Sciatic Nerve Injury in Rats. Frontiers in neuroscience, 2016. 10: p. 589.

32. Zhang, Y., et al., Expression and purification of recombinant human neuritin from Pichia pastoris and a partial analysis of its neurobiological activity in vitro. Applied microbiology and biotechnology, 2015. 99(19): p. 8035-8043.

33. Li, F., et al., MicroRNA-574 is involved in cognitive impairment in 5-month-old APP/PS1 mice through regulation of neuritin. Brain research, 2015. 1627: p. 177-188.

34. Guo, Y., et al., Age- and brain region-associated alterations of cerebral blood flow in early Alzheimer's disease assessed in AßPPSWE/PS1 $\triangle E 9$ transgenic mice using arterial spin labeling. Molecular medicine reports, 2019. 19(4): p. 3045-3052.

35. von Linstow, C.U., et al., Serotonin augmentation therapy by escitalopram has minimal effects on amyloid- $\beta$ levels in early-stage Alzheimer's-like disease in mice. Alzheimer's research \& therapy, 2017. 9(1): p. 74.

36. Vreugdenhil, E. and E. Berezikov, Fine-tuning the brain: MicroRNAs. Frontiers in neuroendocrinology, 2010. 31(2): p. 128-133.

37. Kao, Y.-C., I.F. Wang, and K.-J. Tsai, miRNA-34c Overexpression Causes Dendritic Loss and Memory Decline. International journal of molecular sciences, 2018. 19(8).

38. Wang, X., et al., A Novel MicroRNA-124/PTPN1 Signal Pathway Mediates Synaptic and Memory Deficits in Alzheimer's Disease. Biological psychiatry, 2018. 83(5): p. 395-405.

39. Angelucci, F., et al., MicroRNAs in Alzheimer's Disease: Diagnostic Markers or Therapeutic Agents? Frontiers in pharmacology, 2019. 10: p. 665.

40. Chen, J., et al., MicroRNA expression data analysis to identify key miRNAs associated with Alzheimer's disease. The journal of gene medicine, 2018. 20(6): p. e3014.

41. Fransquet, P.D. and J. Ryan, Micro RNA as a potential blood-based epigenetic biomarker for Alzheimer's disease. Clinical biochemistry, 2018. 58.

42. Harman, M.F. and M.G. Martín, Epigenetic mechanisms related to cognitive decline during aging. Journal of neuroscience research, 2020. 98(2): p. 234-246. 
43. Song, D., et al., miR-199a decreases Neuritin expression involved in the development of Alzheimer's disease in APP/PS1 mice. International journal of molecular medicine, 2020. 46(1): p. 384396.

44. Kang, M.-G., et al., Proteogenomics of the human hippocampus: The road ahead. Biochimica et biophysica acta, 2015. 1854(7): p. 788-797.

45. Han, C.J., et al., Trace but not delay fear conditioning requires attention and the anterior cingulate cortex. Proceedings of the National Academy of Sciences of the United States of America, 2003. 100(22): p. 13087-13092.

46. Webster, S.J., et al., Using mice to model Alzheimer's dementia: an overview of the clinical disease and the preclinical behavioral changes in 10 mouse models. Frontiers in genetics, 2014. 5: p. 88.

47. Hall, J., K.L. Thomas, and B.J. Everitt, Rapid and selective induction of BDNF expression in the hippocampus during contextual learning. Nature neuroscience, 2000. 3(6): p. 533-535.

48. Inagaki, R., S. Moriguchi, and K. Fukunaga, Aberrant Amygdala-dependent Fear Memory in Corticosterone-treated Mice. Neuroscience, 2018. 388: p. 448-459.

49. Blanco-Luquin, l., et al., Early epigenetic changes of Alzheimer's disease in the human hippocampus. Epigenetics, 2020.

50. Sun, M., et al., Cryptic exon incorporation occurs in Alzheimer's brain lacking TDP-43 inclusion but exhibiting nuclear clearance of TDP-43. Acta neuropathologica, 2017. 133(6): p. 923-931.

\section{Figures}


A

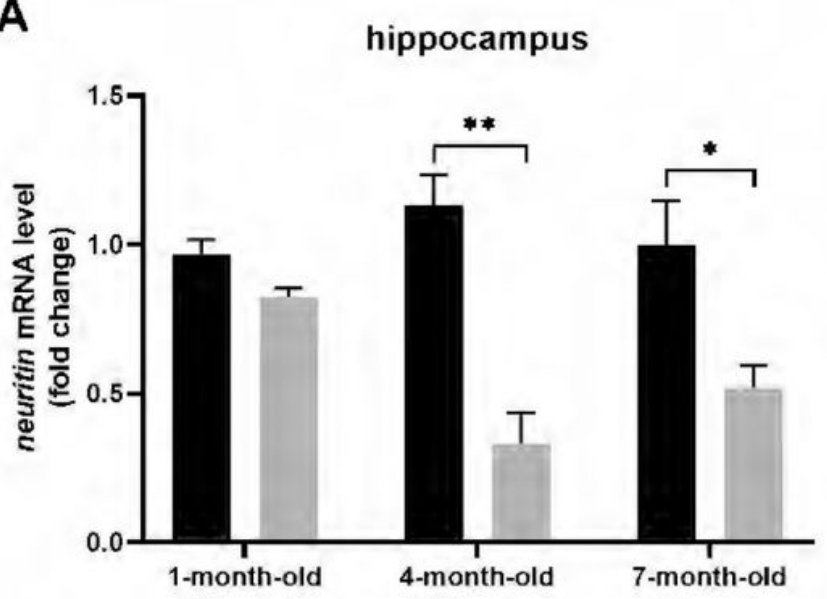

B

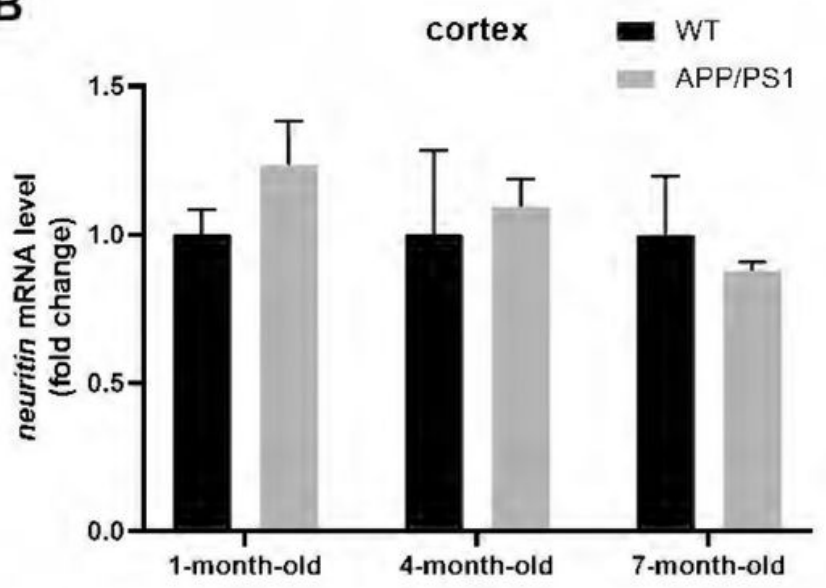

C

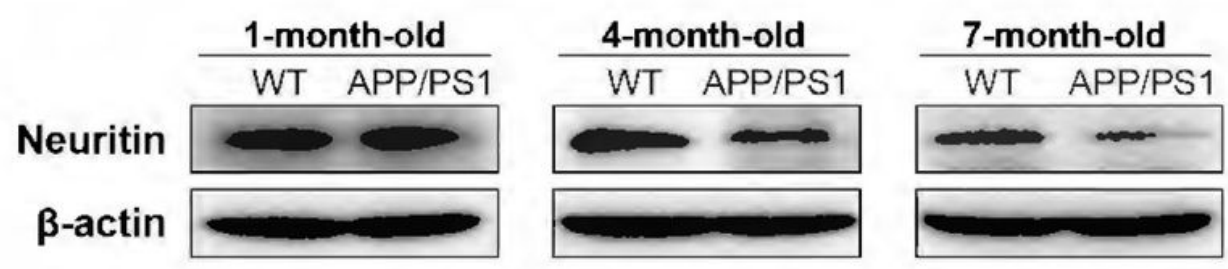

D

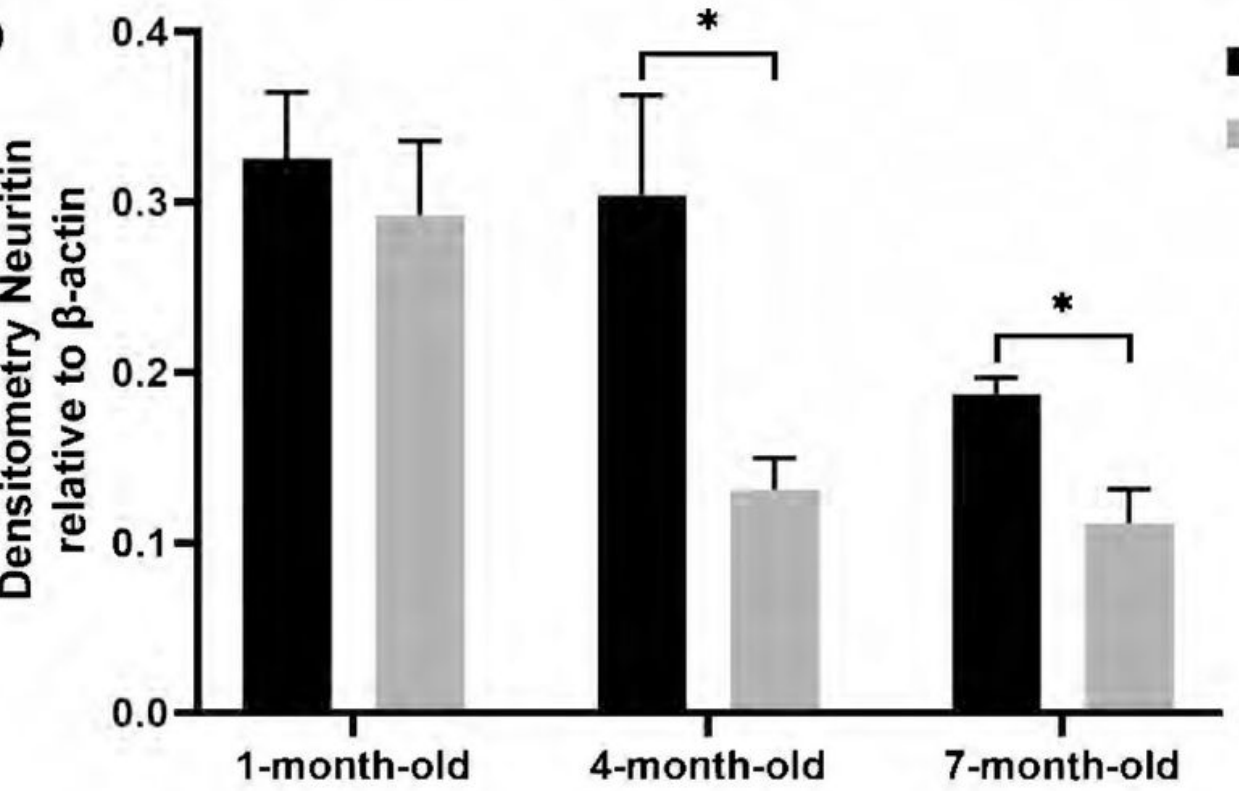

Figure 1

The expression levels of Neuritin mRNA and protein in the hippocampus and cortex of APP/PS1 mice. (AB) Hippocampus (A) and cerebral cortices (B) at the age of 1, 4, and 7 months were obtained from WT mice $(\mathrm{N}=3)$ and APP/PS1 mice $(\mathrm{N}=3)$, and mRNA expression levels of Neuritin was analyzed by RT-PCR. All results were subsequently normalized to GAPDH mRNA level and shown as fold change. (C-D) Protein levels of Neuritin in the hippocampus of WT mice ( $N=3)$ and APP/PS1 mice $(\mathrm{N}=3)$ at the age of 1,4 , and 
7 months, measured by Western bolting assay. $\beta$-actin was expressed as an internal loading control. Statistically, significant differences are denoted by * $P<0.05$, ** $P<0.01$.

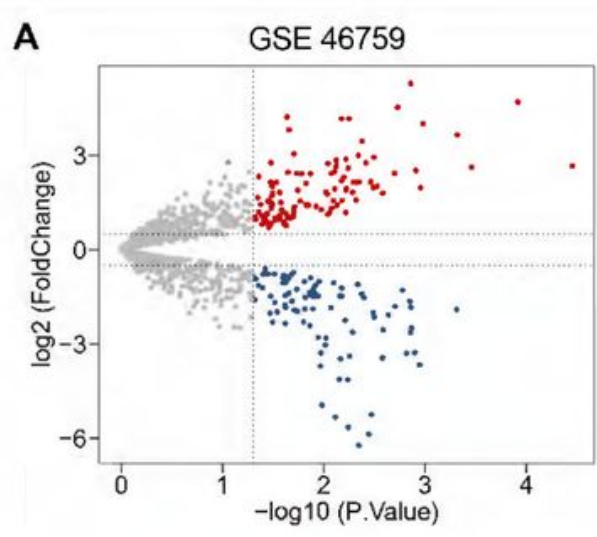

C

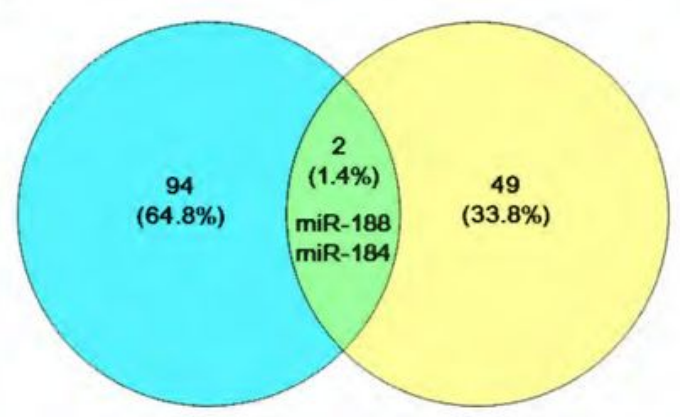

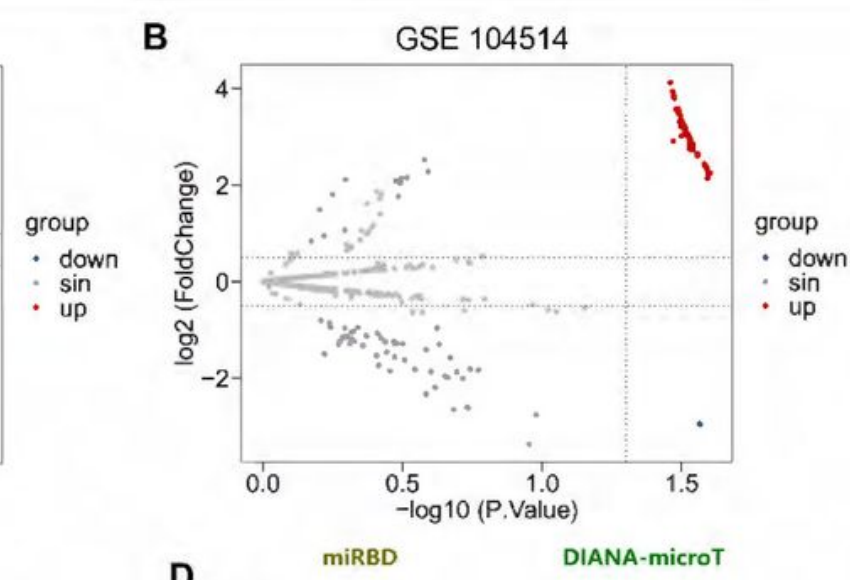

D

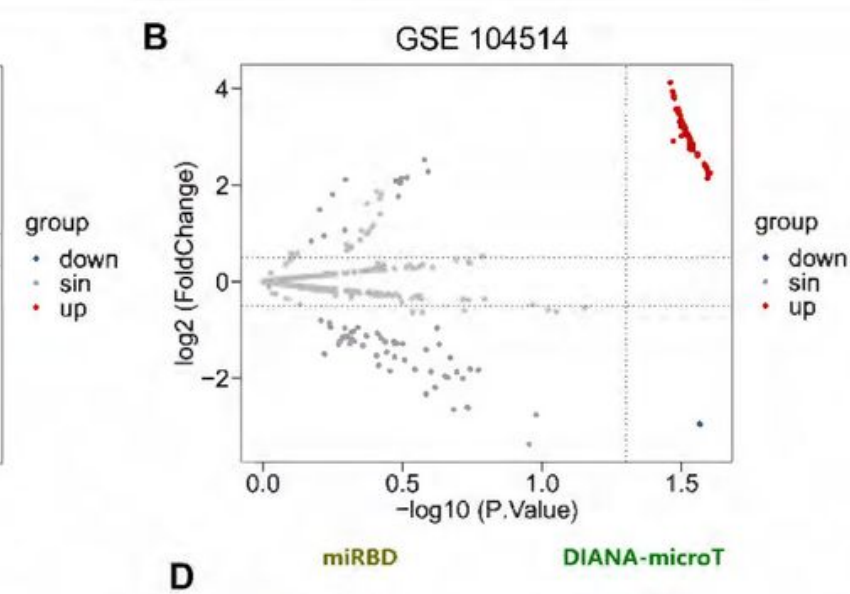

\section{E}
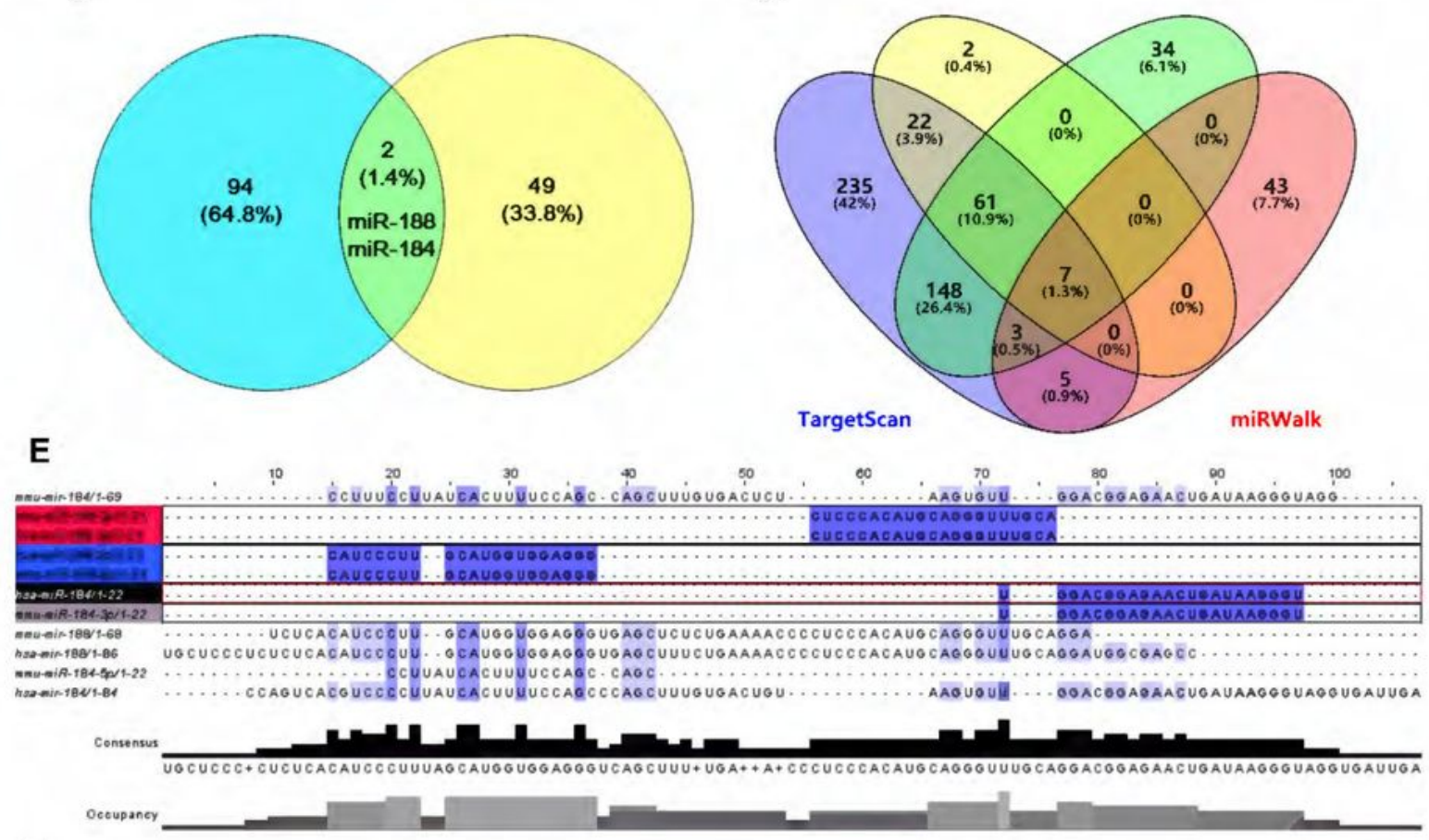

$\mathbf{F}$

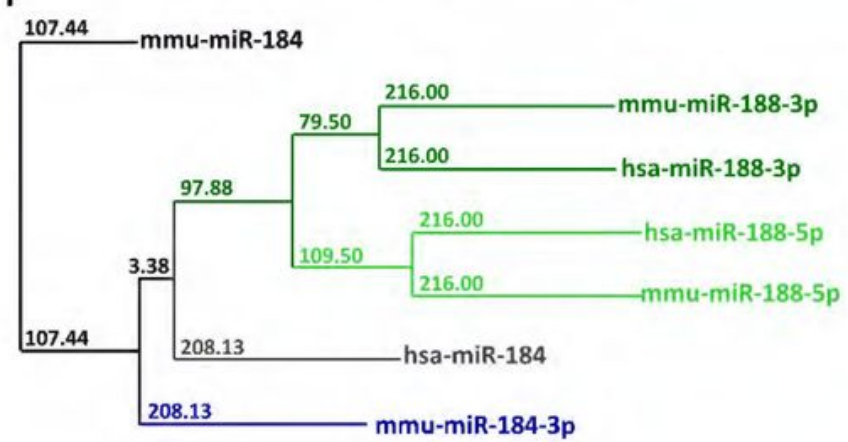

G mmu-miR-188-5p CAUCCCUUGCAUGGUGGAGGG
hsa-miR-188-5p CAUCCCUUGCAUGGUGGAGGG
Consensus CAUCCCUUGCAUGGUGGAGGG

\section{Figure 2}

Screening of candidate miRNAs that may regulate Neuritin expression in AD by Bioinformatics. (A-B) Volcano plots of the miRNAs from cluster analysis of dataset GSE46759 (A) and GSE104514 (B). (C) Venn diagram for overlapping candidate miRNAs from GSE46759 (blue circle) and GSE104514 (yellow 
circle). (D) Venn diagram for overlapping candidate miRNAs that target Neuritin of 4 bioinformatics algorithms, TargetScan (blue circle), miRBD (yellow circle), DIANA-microT (green circle), and miRWalk (red circle). (E) Multiple sequence alignment analysis of candidate miRNAs. (F) Phylogenetic tree construction based on candidate miRNAs. (G) Double sequence global alignment of mmu-miR-188-5p and has-miR188-5p.

A

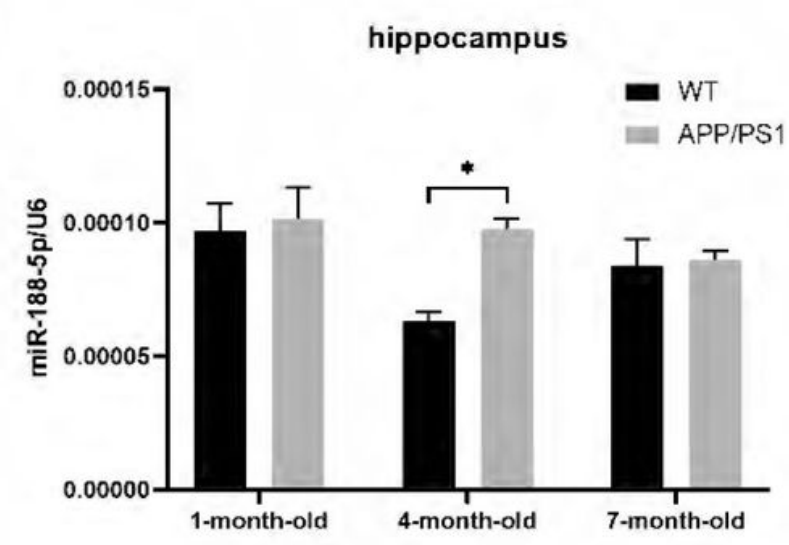

C

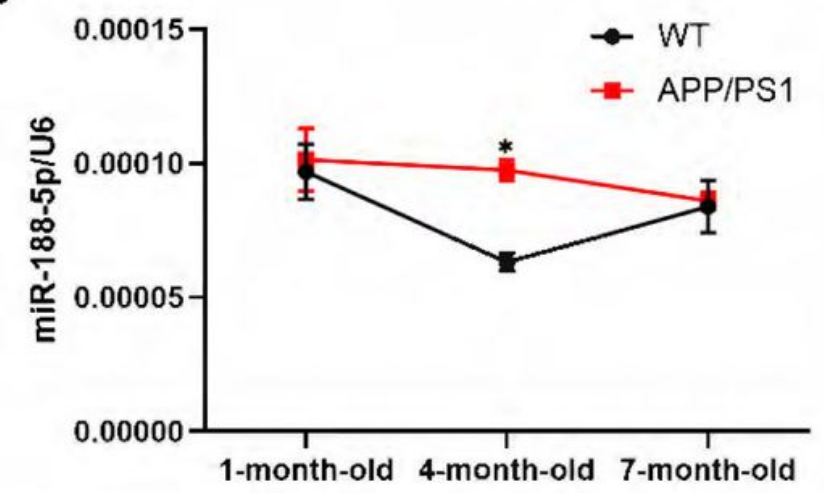

B

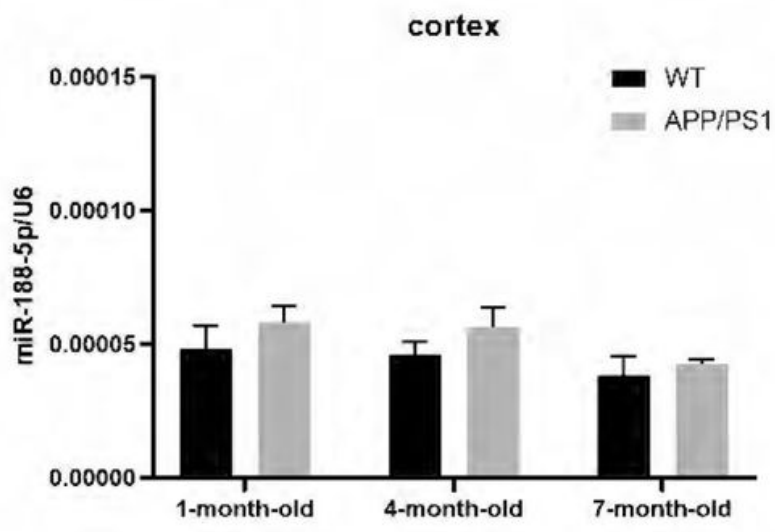

E

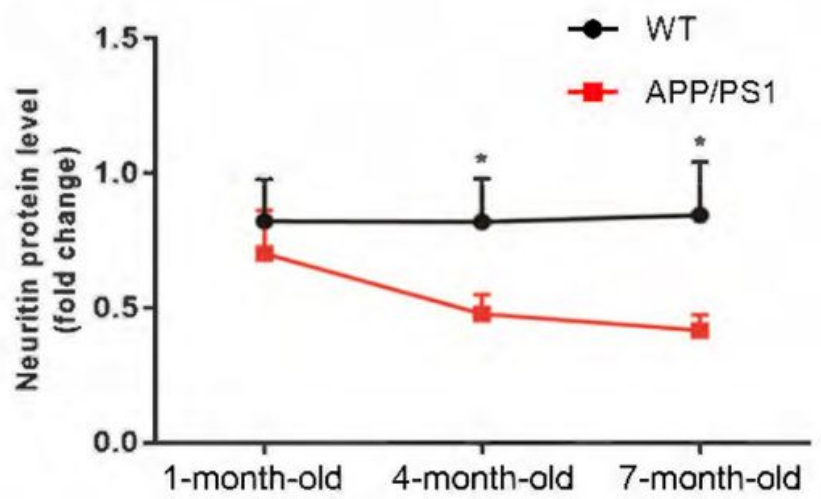

D

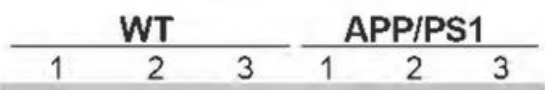

Neuritin

$\beta$-actin

1-month-old

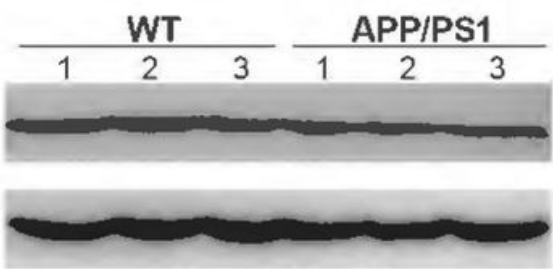

4-month-old

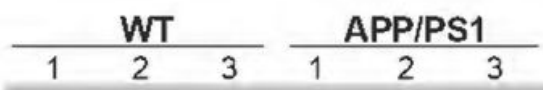

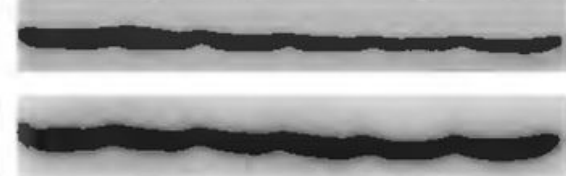

7-month-old

\section{Figure 3}

The levels of miR-188-5p and Neuritin expression in the hippocampus of the APP/PS1 mice. (A-B) The expression levels of miR-188-5p in the hippocampus and cerebral cortex of WT mice $(\mathrm{N}=5)$ and the APP/PS1 mice ( $N=5)$ at the age of 1,4 , and 7 months are measured by quantitative RT-PCR analysis. (C) The expression of miR-188-5p is significantly increased in the hippocampus of 4-months-old APP/PS1 mice. (D) The protein levels of Neuritin in the hippocampus of WT mice $(\mathrm{N}=3)$ and the APP/PS1 mice $(N=3)$ at the age of 1,4 , and 7 months are measured by Western bolting assay. (E) Analysis of the trend 
of the Neuritin protein levels from (D). The expression of $\beta$-actin was used as an internal reference for all results in Western bolting. Statistically, significant differences are denoted by * $P<0.05$.
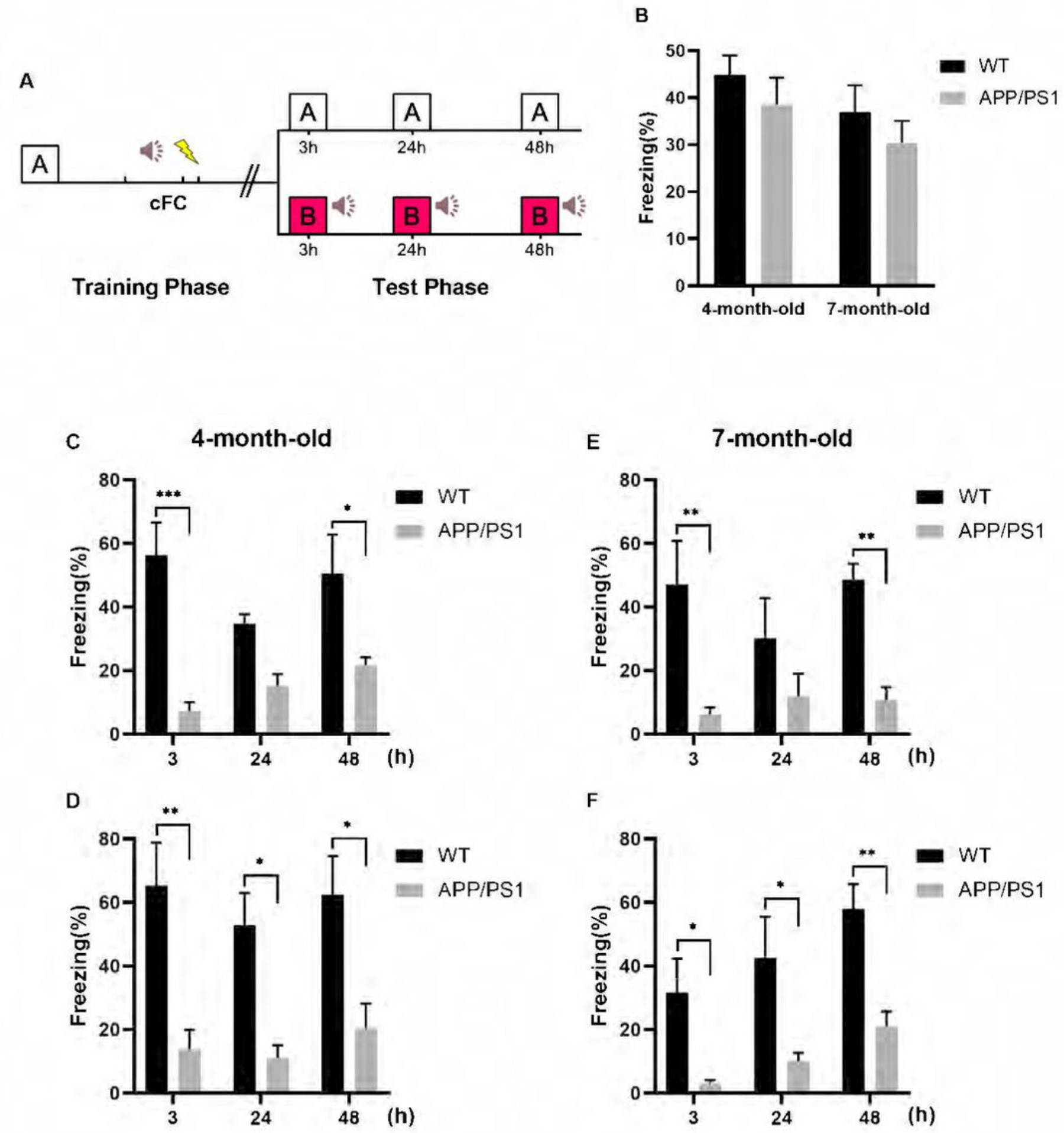

Figure 4

The manifestations in the Fear Conditioning test of APP/PS1 mice. (A) Schematic diagram of the Fear Conditioning test. (B) The freezing percentages of 4- and 7-months-old APP/PS1 mice ( $\mathrm{N}=5$ per age cohort) during the training phase, compared with age-matched WT mice ( $\mathrm{N}=5$ per age cohort). (C-F) The 
changes in freezing percentage of 4- and 7-months-old APP/PS1 mice during the contextual (C, E) and cued (D, F) memory tests of the test phase, compared with age-matched WT mice. Statistically significant differences between groups are denoted by * $P<0.05$, ** $P<0.01$, *** $P<0.001$.
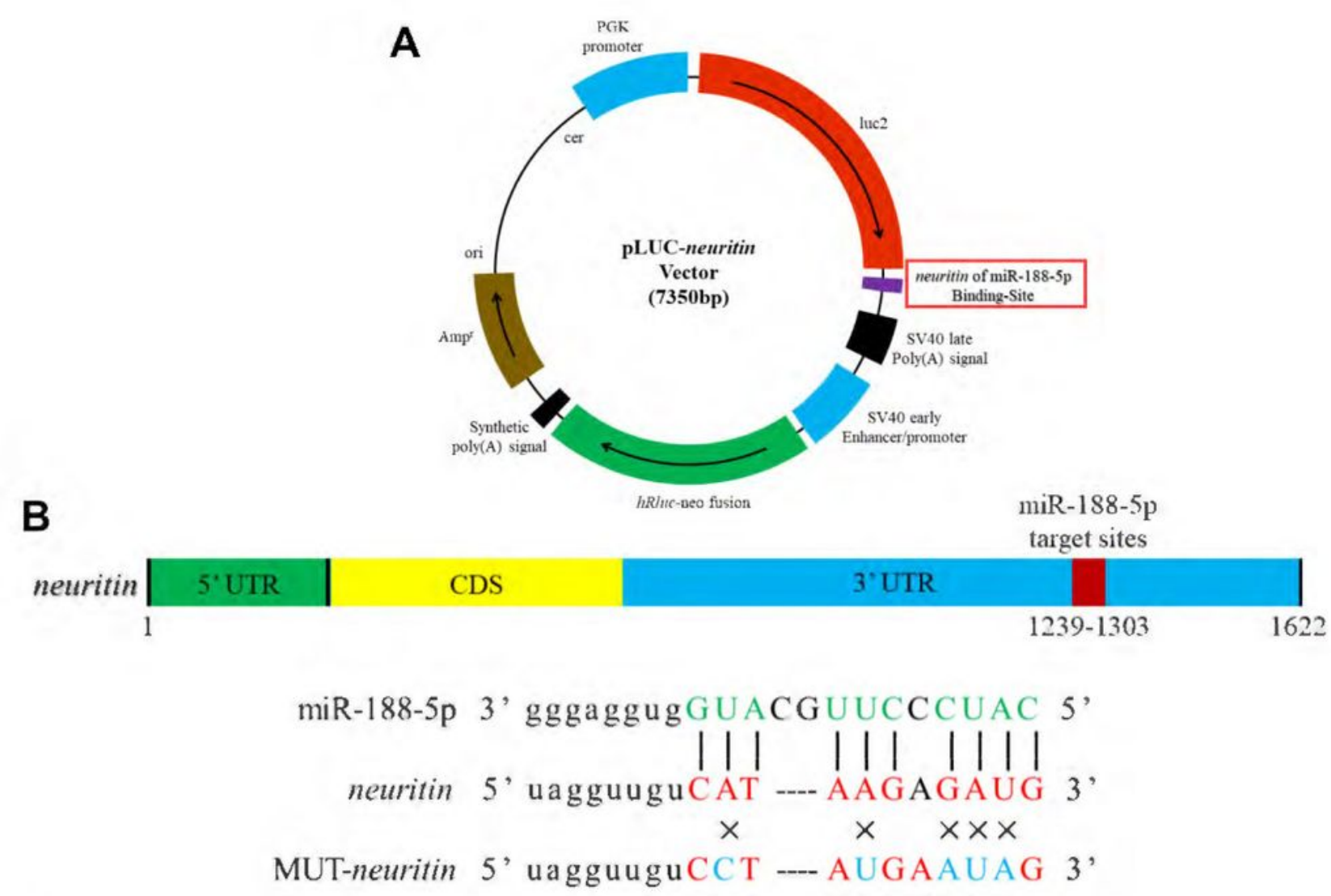

C

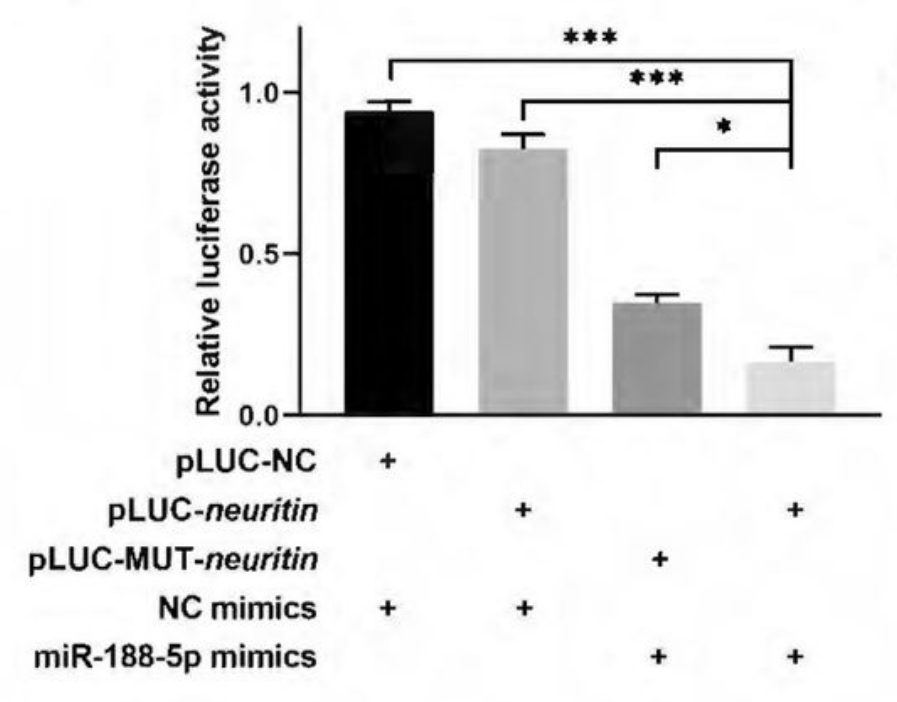

D

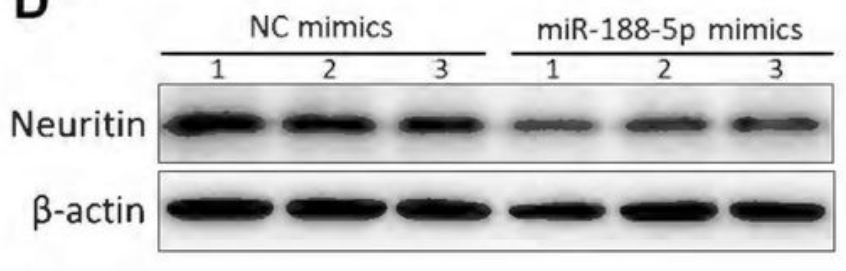

E

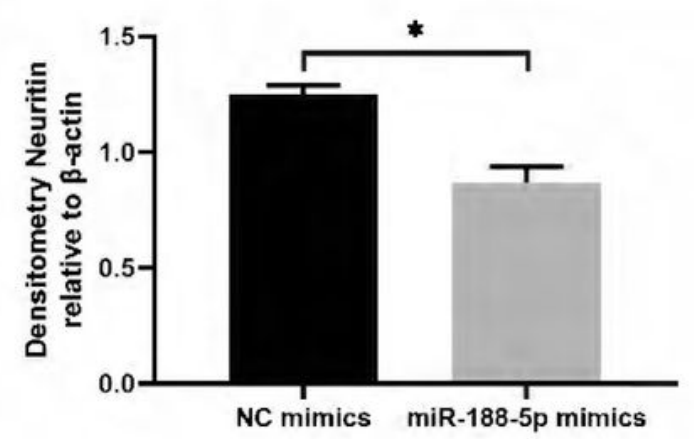

Figure 5

The regulation of miR-188-5p targets Neuritin in 293T cells. (A) The schematic diagram of the p-LUCneuritin vector. (B) The binding sites of miR-188-5p in the 3'-UTRs of neuritin and MUT-neuritin. (C) The 
luciferase reporter activity assay in 293T cells transfected with miR-188-5p mimics and co-transfected with pLUC-NC, pLUC-neuritin, and pLUC-MUT-neuritin. (D, E) The expression levels of Neuritin protein in miR-188-5p mimics transfected 293T cells measured by Western blot analysis. Statistically significant differences between groups are denoted by * $P<0.05$, ** $P<0.01$, *** $P<0.001$. 